\title{
Relationship analysis from the perspective of interpersonal Pragmatics
}

\author{
Li Lan \\ Humanities School, Wuhan Guanggu vocational college, Wuhan, China
}

Keywords: interpersonal pragmatics; interpersonal relationship; new development direction

\begin{abstract}
: with the development and progress of the society, people gradually began to pay attention to the interpersonal relations from the angle of pragmatics, the study of interpersonal relationships to bring new development opportunities and to a certain extent. The author first analyzes and explores from the perspective of interpersonal pragmatics, and effectively improves the research direction of interpersonal relations. The research is divided into three directions. First, it discusses the pragmatic features of interpersonal relations, and the relationship between interpersonal communication and face, politeness and emotion. Second, it can be carried out in terms of interpersonal and analysis of interpersonal relations between language, explore effective, study of the relationship of dimensions of the main purpose is to according to the traditional interpersonal language to the interpersonal language in the research study and research contents of language further improve interpersonal dimension. In order to more effectively promote future studies.
\end{abstract}

\section{Introduction}

In the early years, some western developed countries have made a certain evaluation of the face in interpersonal communication. Face mainly refers to the number of positive social evaluation to some social contacts in other given. To a certain extent, it can bring multiple and bidirectional social interaction, so that it can bring certain interpersonal influence to the society, and can further influence the research of interpersonal pragmatics. "Face" is a representative of politeness thought, which can effectively deal with some dimensions of interpersonal relationship involved in face and politeness, but to a certain extent, lack of specialized research and exploration. And there is no separate analysis and topic establishment, so it is not fully embodied in the process of interpersonal communication pragmatics characteristics and related forms of expression [1].

Study on the interpersonal pragmatic stage gradually got everyone's attention, and many studies have noted the pragmatic interpersonal problems, and new research topic and research put forward timely claims. The research and analysis of gradually, people have gradually developed a variety of voice and patterns of various linguistic resources, effectively establish a certain relationship, and do the corresponding maintenance, so it can effectively improve some problems in interpersonal relationship in the pragmatic study. Therefore, the relationship between interpersonal pragmatics is not a mere contextual factor, but an independent research object, which can reflect a process in communication [2].

\section{Dynamic pragmatic features in interpersonal relationships}

According to the relationship in the process of interpersonal communication at this stage, we can see that we now have two important understanding of the relationship analysis from the perspective of interpersonal learning. First of all, we can regard the interpersonal relationship as a contextual factor and contextual dimension, and there is a cumulative and discrete result, and all of these have certain process phenomena. When there are some dynamic pragmatic features in interpersonal relationships, the main feature of interpersonal communication is to explore a kind of understanding and effect of interactive communication. At this stage, a lot of attention should be paid to communicators to effectively improve the concept of self, as well as some personal cognitive experience and some adjustments to interpersonal relationships. 
And at this time, some for some relationship in interpersonal pragmatics can see some processes, and can effectively carry out some non accumulation of behavior, and is able to construct and embodied in the communicative interaction. So once we put the interpersonal pragmatic as a process, so we should explore and communicate effectively, and can use the language effectively and non linguistic resources, and to build a relationship between communicative subjects, which will reflect the dynamic relationship between communication subject and to a certain extent. So, the interpersonal relationship has certain subjectivity, are the interactive results of dynamic communication between subjects, a cause of interpersonal relationship is also one of the communication, and the manners and speech communicative behavior is appropriate is a process of communication success conditions. The corresponding research shows that interpersonal relationship is actually a key part of communicative interaction, which can effectively form the social contact between people.

On the interpersonal communication of them there are some different understanding, and this knowledge will affect the interpersonal communication activities of people in a certain extent, and will affect the people involved in the process of communication on the relationship and some related concepts. In the process of interpersonal communication, there are many links with interpersonal relationships, such as harmonious management, relationship work and relationship building. They are in a certain extent can be seen as a context, and that is the defect of interpersonal relationship between the politeness principle and the face theory, instead of its research and analysis from the perspective of more widely. In the study of face and politeness, the traditional sociolinguistics and pragmatics have been removed, but the dynamic phenomenon has not been shown yet. That is to say, a relationship between the contextual factors under the terms of the interpersonal perspective is not the traditional static, but can be a phenomenon to study object, the relationship can also be studied and analyzed in the interpersonal interaction, summed up the changes and some [3].

\section{Interpersonal relations reflected}

At this stage, because we always think of the interpersonal relationship in interpersonal pragmatic mainly refers to the human face and politeness perspective to analyze, in interpersonal communication, some people will be able to face and politeness and the relationship is regarded as a kind of interpersonal communication can be intertwined, but some researchers believe that at this stage they to be able to reflect on the same level. Face is to communicate a staff relative to other people need to be carried out. Some people think that the face of the present and acceptable way is actually a work, and is mainly to see how communication personnel selection, and to see how to start, and then to be able to construct a kind of identity. Therefore, after many studies and analysis, people can make personal contacts according to face and politeness and other related social communication terms, and relate to the judgment of the relationship. At present, many traditional pragmatics can effectively explore the nature of language, and can not be able to some other interpersonal resources except language. In addition to some traditional hungry language resources and social and cultural resources, a lot of body language and some gestures in the process of speaking are the object of interpersonal relationship research [4].

\subsection{Interpersonal relationship in face study}

When people face some face problems, they usually use static and gradual cumulative perspective, and can regard interpersonal relationship as a contextual factor. Although the study of face and relationship in the past can continue to deepen its research, but there are some essential problems in the understanding of the relationship. The word "face" was first appeared in our country, after the study of western developed countries concerned, speech acts in the face of the interpersonal relationships in a more expanded, and effectively affect the pragmatic dimension.

Face is actually necessary for people in the normal social needs. Face is a dominant factor in interpersonal communication, and the relationship is only an important interpersonal communication language which can affect the face. At present, many researchers have defined some 
positive properties involved in interpersonal communication, and in the process of interpersonal communication, the relationship between the management has become the people in the study of interpersonal relationships summary, put forward the framework of the eyes more effectively to a certain extent. Because it not only puts forward a more effective and positive relationship dimension, but also includes some information related factors. According to this kind of acting and analysis, not only can effectively deepen the relationship between people in interpersonal relations, but also can effectively carry out the research and analysis of the relationship between the pragmatic attributes.

But the research in many relationships in the language of our country at this stage is not enough by building relationships, the dynamic characteristics and the lack of effective communication in the main embodiment, the excessive emphasis on some personal thoughts. In daily life, interpersonal interaction in communication from the perspective of the face is regarded as a kind of relationship phenomenon, and researchers believe that the face is actually related to an individual interpersonal relationship in understanding. Effective understanding face in interpersonal relationship is an important factor in the success of interpersonal communication.

\subsection{Interpersonal relationships in politeness studies}

Study of the most important research on politeness is the interpersonal relationship. In the traditional sense, politeness research is mainly based on English related culture as the main research environment, and can analyze the face and politeness analysis, find out the principles of politeness and related principles, etc. In the study of politeness principle, some researchers have regarded maintaining harmonious interpersonal relationship as an important communicative goal. And to recognize the pragmatic ability of rules, and can be directly defined as a polite maintenance definition of interpersonal relationship, can reduce the maximum degree of some contradictions in interpersonal communication, the cooperative principle to establish some basic principles and related countermeasures, and points out that the. In the study of interactive principles such as social communication, some people believe that it mainly involves some more urgent and deep-seated social, and it is an effective pragmatic constraint in interpersonal communication. If in interpersonal relationships in the society do not comply with these principles, then there will be some problems.

\subsection{Interpersonal relationships in emotion research}

The emotional impact is very important in interpersonal communication, and after a long time to carry out a pragmatic study of effective, and there is no influence of some communicative process in the interaction of emotion and effective attention to interpersonal relationship. If we can combine emotion, emotion and area effectively, we can cultivate good sense of communication. From the perspective of interpersonal analysis can be seen, the two section points out that successful interpersonal interactions in interpersonal communication in the open to emotional information, and can not balance the interpersonal relationship, and to a certain extent, the interpersonal relationship is polite and courteous emotional communication [5].

\section{The choice of research objects in interpersonal relationship}

In interpersonal pragmatics, in the process of interpersonal interaction, a lot of language and voice and emotion are jointly hungry, and also involves the expression of many relationships and the establishment of ideas. The important purpose is to convey the content and the corresponding information better. Therefore, in the study of interpersonal pragmatics itself, it can be seen that many people who can even provide interpersonal evidence and clues are the objects of interpersonal language research. It is mainly concerned with interpersonal language and some nonverbal things in the study of interpersonal pragmatics. And in the process of the study of the relationship, the choice of the scope of the sentence is mainly based on some basic conditions for analysis. First, the interaction in social communication is interpersonal, and should occur in more than two people in the space, some languages mainly include the direct or indirect to participate in the object. Second, the identity of the participants, can effectively observe some of the relationship between the 
establishment and change. Third, the information content of communication has certain interpersonal characteristics, can be the characteristics of the mind and the corresponding emotional attitudes, etc., and should limit the factual information transmission. Fourth, the way of communicating information can communicate with some languages, and also can improve some communicative modes of calculation. The object of study can be regarded as a discourse as well as some behavior or some form of body language and communication. Like some small movements of some people living in the small eyes and are able to convey some intention. And these communicative behaviors and states can express certain interpersonal emotions, attitudes and emotions, and can effectively improve the communication relationship between people [6].

\section{Conclusion}

According to the people according to different communication environment and communicative object can effectively establish some dynamic performance, and increase in pragmatic research can be gradually hungry, and can show that interpersonal communication in the relationship dimension. In the relevant concepts, IQ can show obvious characteristics, so that in jumping out of the traditional interpersonal language state, can effectively analyze the relationship between face and politeness and emotion. It is more effective to improve the development of interpersonal relations when the relationship is regarded as a relatively independent research thinking. It is believed that this research and analysis can be helpful to the analysis of interpersonal relations from the perspective of interpersonal pragmatics.

\section{References}

[1] Grainger K. Of babies and bath water: Is there any place for Austin and Grice in interpersonal pragmatics [J]. Journal of Pragmatics, 2013, 58(58):27-38.

[2] O’Driscoll J. The role of language in interpersonal pragmatics[J]. Journal of Pragmatics, 2013, 58(52):170-181.

[3] Locher M A. Interpersonal pragmatics and its link to (im)politeness research[J]. Journal of Pragmatics, 2015, 86:5-10.

[4] Ran Y, Liu P. The Study of Relation from the Interpersonal Pragmatic Perspective[J]. Foreign Language Education, 2015.

[5] Svetanant C. Acknowledging a debt of gratitude in Japanese Persuasive Discourse: An Interpersonal Pragmatic Perspective[J]. Internet Journal of Language Culture \& Society, 2016(42):54-70.

[6] Arundale R B. Face as a research focus in interpersonal pragmatics: Relational and emic perspectives[J]. Journal of Pragmatics, 2013, 58(58):108-120.

Lan Li Female, Hubei province, April 4th, 1980. Master degree, foreign linguistics and applied linguistics. 